\title{
Evolutionary dynamics for the generalized Baliga-Maskin public good model
}

\author{
Elvio Accinelli ${ }^{\mathrm{a}}$, Filipe Martins ${ }^{\mathrm{b}, \mathrm{c}, *}$, Alberto A. Pinto ${ }^{\mathrm{b}, \mathrm{c}}$ \\ a Facultad de Economía de la UASLP, México \\ ${ }^{\mathrm{b}}$ Departamento de Matemática, Faculdade de Ciências da Universidade do Porto, Portugal \\ ${ }^{\mathrm{c}}$ LIAAD-INESC, Porto, Portugal
}

\section{A R T I C L E I N F O}

\section{Article history:}

Received 31 March 2019

Revised 18 October 2019

Accepted 19 October 2019

Available online 30 October 2019

\section{Keywords:}

Common and public goods

Externalities

Free-riding

Evolutionary dynamics

Adaptive dynamics

Stability

Environment

Pollution.

\begin{abstract}
A B S T R A C T
The problem of the consumption or provision of common and public goods is a well known and well studied problem in economic sciences. The nature of the problem is the existence of non-excludable externalities which gives rise to incentives to free-riding behaviour. There are several economical frameworks trying to deal with the problem such as coalition theory or mechanism design and implementation theory to ensure a Pareto efficient consumption or provision of such good. Baliga and Maskin considered an environmental game where several communities face a problem of pollution reduction. They show that all communities except one of them have incentives to act as a free-rider, i.e. only one community is willing to face the costs that air cleaning implies, namely the one with greatest preference for the good. In this work we introduce an adaptive evolutionary dynamics for the generalization of the BaligaMaskin model to quasi-linear utility functions. We show that the Baliga-Maskin equilibrium is the only asymptotically stable dynamical equilibrium, all others being unstable. This result reasserts the problem of free-riding and externalities for the case of a common good in a dynamically/evolutionary setting, and reiterates the relevance of mechanism design and coalition formation in the context of dynamical models.
\end{abstract}

(c) 2019 Elsevier Ltd. All rights reserved.

\section{Introduction}

In economic sciences when a group of economic agents consumes or makes a provision for a public good or a common good, normally some problems arise when trying to do it efficiently, either because the good is over-consumed or under-provided, resulting in negative externalities affecting all agents, i.e. non-excludable externalities. Usual examples include exhaustion of fish or coal stocks for the case of common goods or common-pool resources, and air or sea pollution related to the overuse of air and water resources in the case of public goods. The reason for this inefficiency lies in the fact that common or public goods are non-excludable goods, i.e. a good whereby it is not possible to efficiently exclude people from using or having access to the good, even those that have not paid for its consumption or contributed to its provision. According to the usual definitions, a public good is a good that in addition to being non-excludable is also non-rivalrous or nonsubtractable, meaning that consumption by one agent does not reduce the ability of another agent to consume the good, thereby

\footnotetext{
* Corresponding author.

E-mail addresses: philip_m90@hotmail.com (F. Martins), aapinto@fc.up.pt (A.A. Pinto).
}

making it difficult to restrict access to the good based on price since the marginal cost of providing the good to an additional individual is zero. Non-excludability causes individuals to have incentives to act as "free-riders", since if they do not pay for such a good or do not contribute to its provision, they will not be excluded from its using. Such behaviour is possible if there are other groups of individuals who are willing to pay the cost or make the effort that the provision of the common or public good requires, in which case an individual agent may be tempted to "free-ride" on the effort made by the others in the sharing of the costs of consumption or provision. The obvious social consequence is that successive free-riding may cause a lowering of the provision of the good, or alternatively over-consumption due to not all consumers contributing to its maintenance. Several solutions for the problem have been considered in the past such as the so-called Coasian position arguing that given an inefficient allocation it leads to incentive to bargain in the direction of a Pareto-optimal or efficient allocation. The problem of free-riding and externalities related to the difficulties of the governance and administration of common and public goods have been widely studied. For instance, we mention the seminal studies $[7,8]$.

In [6] the authors propose a model for the consumption and provision of a public good in the case of environment protection. 
They consider a non-excludable externality, which in their case is air pollution, and the problems of mitigation of such externality which will affect any agent, even those that do not pollute. In their case the public good is the reduction of air pollution itself which is non-excludable since any agent will benefit from the reduction of pollution, which nonetheless bears a cost and hence causes incentives to free-riding. In a broader context the nonexcludable externality may be sea pollution or more generally be the overuse of a common-pool resource such as natural resources as fish stocks or forests, or the under-provision of a public good such as national defence, and the problem of their sustainable preservation. The model may also be seen more generally as a model for the contributions to a good that is non-excludable, i.e. a common or a public good, with the decision variables being the effort or amount of contribution. Their results show that when several communities or economic agents are interested in the contribution or consumption of a non-excludable good (in the case considered, the good is the reduction of pollution), Coasian bargaining (i.e. bargaining in the presence of externalities) by the agents will not lead to a Pareto-efficient outcome. This is due to the non-excludable nature of the externality, be it positive, as a public good, or negative. Indeed, when all communities are participating in an agreement, each community will be better off by not contributing, hence free-riding on the others efforts ending up in a non Pareto-optimal solution, and with each agent or community contributing its individually optimal level which falls short of the total joint contribution for the Pareto-optimal solution. More precisely, the community with the greatest preference for the maintenance of the good, in the case, the reduction of air pollution, will alone bear the costs associated to the reduction. This solution may be seen as a Pareto-dominated Nash equilibrium for a two population normal form game.

In this work we introduce a dynamics, the so called adaptive dynamics from the theory of evolutionary games (see Hofbauer and Sigmund [3] and Sigmund [9]) to a generalized version of the common good contribution/pollution reduction model in [6]. Adaptive dynamics was first introduced in the context of evolutionary game theory to address the problem of the direction of evolution given a certain fitness function, pointing towards the most favourable direction of evolution. Adaptive dynamics has been increasingly used in contexts other than biology such as economics and other social sciences. We shall show that in a generalized version of the environment protection model of Baliga and Maskin the equilibria for the adaptive dynamics are the equilibria where one community alone is bearing the costs of maintenance of the good, while the others are free-riding on this effort. In our generalized version we consider quasi-linear utility functions and we assume heterogeneity regarding the marginal utilities between the communities involved in the common good game. Furthermore, the stationary states of the dynamics are all unstable with the exception of one equilibrium, which is the one where only the agent with greatest preference (or more generally, the one with the highest marginal gross benefit) contributes.

The results in our work can be seen as an evolutionary dynamical reassertion of the results by Baliga and Maskin in the sense that it raises the problem of sustainability of the exploration and provision of common goods such as the question of contribution for the reduction of pollution in an evolutionary framework. In their paper they argue that to ensure the Pareto-optimal allocation one needs to consider intervention of some sort providing a mechanism so that this outcome can be attained in a stable way. This is the goal of implementation theory or the theory of mechanism design. See for instance [5] for a survey on some of the main features and results of mechanism design theory. Our results thus highlight also the importance of evolutionary stability in addressing of problems of the governance of common and public goods where the issue of free-riding appears and the implementation of social goals regarding their management. See for instance [1] for an economic application of adaptive dynamics in the framework of mechanism design and implementation theory and [4] for how evolution can help solve the problem of the tragedy of the commons. Apart from the study of mechanisms enforcing contributing to the solution of problems of governance of the commons, there are alternative approaches such as clubs or coalition theory approach whose relevance in cases of climate policy and free-riding have been pointed in works such as [2] where coalitions may form that share the costs of the provision of the goods, such as the costs of reducing air or sea pollution. See [8] for more on the importance of coalitions for questions regarding common goods. The question of game theoretic stability as well as evolutionary dynamical stability of such coalitions are essential to the study of the problem, together with the study of the conditions in which it is possible to render them stable.

This work is organized in the following way. In Section 2 we introduce the common good game inspired by the air pollution reduction game of Baliga and Maskin [6]. We describe the utility functions we consider and discuss the assumptions we make on such utilities regarding the heterogeneity of the communities involved, and we propose and describe the evolutionary adaptive or myopic dynamics for the game. In Section 3 we obtain our results regarding the stability analysis of the equilibria of the evolutionary dynamics for the generalized version of the Baliga Maskin game described in the previous section. We present some conclusions summarizing our results and several possibilities of future research in related topics in Section 4.

\section{Evolutionary dynamics for the common good model}

Following [6], we consider an example based on environment contamination, where $N$ communities (labelled $i=\{1, \ldots, N\}$ ) would like to reduce their aggregated polluting emissions. Denote by $r_{j} \geq 0$ the reduction of pollution by community $j$ and by $r=\sum_{j=1}^{N} r_{j}$ the aggregated pollution reduction. Given a vector $\left(r_{1}, \ldots, r_{i}, \ldots, r_{N}\right)$ of individual contributions (i.e. reductions of pollution) we will denote by $r_{-i}=\left(r_{1}, \ldots r_{i-1}, r_{i+1}, \ldots, r_{N}\right)$ the contributions of every community except community $i$. In a broader context the variables may be referred to as the contributions for the common good. In the context of air pollution as the nonexcludable externality, the common good is the reduction of pollution and we will refer to the variables as the contributions or effort for the reduction of pollution by the communities involved.

Suppose that the utility to a community $j$ is given by a quasilinear utility function of the form:

$\mathcal{U}_{i}\left(r_{i}, r_{-i}\right)=u_{i}(r)-\beta_{i} r_{i}$.

depending on the individual contribution $r_{i}$ and also on the contributions of the other communities $r_{-i}$. As usual for quasi-linear utilities, the function $u_{i}$ is assumed to be strictly concave on the aggregated contribution $r$ and $\beta_{i}>0$ is the marginal cost. The functions $u_{i}$ are the gross benefit of the reduction of pollution. In most situations we have $u_{i}(r)=u\left(r ; p_{i}\right)$ for some parameter $p_{i}$ characterizing community $i$. We will denote the marginal benefit of community $i$ by $v_{i}(r)=\partial u_{i} / \partial r$. Strict concavity implies that $v_{i}$ is strictly decreasing on $r$, i.e. $\partial v_{i} / \partial r<0$.

For the adaptive dynamics analysis in the next section, we require two more assumptions on the marginal benefits. We will assume that

$\lim _{r \rightarrow 0} v_{i}(r)>\beta_{i}>\lim _{r \rightarrow+\infty} v_{i}(r)$.

We observe that the usual Inada conditions for the gross benefits $u_{i}$ imply the above conditions. 
Furthermore we will assume that the marginal benefits are different for each community. More precisely, we will assume that for every community $i$ we have:

$v_{i}(r)>v_{i+1}(r)$.

In other words, we assume heterogeneity in the marginal gross benefits of the communities.

Example. Consider the following modification of the BaligaMaskin utility function where the gross benefit $u_{i}(r)$ is given by $\theta_{j} r^{\alpha}$, where $\alpha \in(0,1)$ is the intensity of reduction and $\theta_{j} \in \mathbb{R}^{+}$, for $j \in\{1, \ldots, N\}$ are the preferences of community $j$ for clean air. This gross benefit is strictly concave since $\alpha$ is less than 1 and so the utility function given by $\mathcal{U}_{i}\left(r_{i}, r_{-i}\right)=\theta_{j} r^{\alpha}-r_{j}$ is quasi-linear.

Furthermore, the marginal benefit for community $i$ is given by $v_{i}(r)=\alpha \theta_{i} r^{\alpha-1}$. This function is monotonically increasing in $\theta$ and these utility functions satisfy the previous assumption if the preferences $\theta$ are such that

$\theta_{1}>\theta_{2}>\ldots>\theta_{N}$.

These inequalities mean that the individuals or communities involved in the pollution of the environment have different preferences for clean air, and then different incentives to cooperate for the clearing of the air.

We consider the adaptive or myopic dynamics (see for instance Hofbauer and Sigmund [3], Sigmund [9] and Cabrales [1]) for the game "free-riders $v s$. cooperation":

$\dot{r}_{i}=\frac{\partial \mathcal{U}_{i}}{\partial r_{i}} r_{i}, \quad i=1, \ldots, N$.

where the phase space is the non-negative orthant of $\mathbb{R}^{N}$. We observe that the vector field defining the system of differential equations might blow up at the origin and be undefined.

The reason for the nomenclature myopic is that in each state of the dynamics, i.e., each state of the contribution for the public good, agents change their contribution in the direction which is the most promising one according to their marginal utility.

\section{Stability analysis of the myopic dynamics of the effort}

In this section we present the stability analysis of the dynamics introduced in the previous section.

Consider the utility function of the $j$ th community given by $u_{j}(r)-\beta_{j} r_{j}$ as previously described with the assumptions we made, namely strict concavity of the gross benefit $u_{j}(r)$ and heterogeneity regarding the marginal benefits $v_{j}(r)$. We will assume some homogeneity in the costs $\beta_{j}$, i.e. we will assume that the costs are relatively similar, i.e. $\beta_{j} \approx \beta$ and without loss of generality we will assume that the marginal costs are $\beta_{j}=\beta=1$. The "myopic" dynamics is given by the system of differential equations:

$\dot{r}_{j}=\frac{\partial \mathcal{U}_{j}}{\partial r_{j}} r_{j}=\left(v_{j}(r)-1\right) r_{j}, j=1, \ldots, N$.

According to this dynamics each community increases its contribution/reduction when its utility increases, and conversely decreases its contribution when the utility decreases and it does so in a per capita rate equal to its marginal utility.

We first observe that the coordinate axes $r_{j}=0$ are positively invariant for this dynamics as well as the interior of the nonnegative orthant of $\mathbb{R}^{N}$. The marginal utilities are given by:

$\frac{\partial \mathcal{U}_{j}}{\partial r_{j}}=v_{j}(r)-1$

For each of the differential equations composing the system (2) the right-hand side is zero when $r_{j}=0$ or when $v_{j}(r)=1$. By strict concavity of the gross benefit $u_{i}(r)$ we have that the marginal benefit $v_{j}(r)$ is a strictly decreasing function and so the previous equation has a unique solution $\bar{r}_{j}$, i.e. such that $v_{j}\left(\bar{r}_{j}\right)=1$.

When the aggregated contribution $r$ is such that $r=\bar{r}_{j}$ then the $j$ th community is offering the effort that according to their preferences corresponds to their optimal contribution, i.e. the effort that maximizes his/her utility. In this case, the time derivative $\dot{r}_{j}$ is zero, so $j$ does not change its contribution, although the aggregated contribution might change due to changes of the other agents. We observe that if $r>\bar{r}_{j}$ then the agent decreases its contribution whereas if $r<\bar{r}_{j}$ he/she increases its contribution. This is the main idea of the aforementioned adaptive dynamics where agents locally optimize their strategy in the direction which grants higher utility levels. Hence we may call $\bar{r}_{j}$ the aggregated contribution threshold for community $j$ : it is the aggregated contribution level that must be achieved in order that $j$ does not need to increase its contribution, or in other words it may be regarded as the subsistence aggregated contribution for $j$.

With the previous assumptions on the marginal utilities of the communities we have heterogeneity of the agents in the sense that the aggregated contribution thresholds are such that $\bar{r}_{i}>\bar{r}_{i+1}$. Taking this into account the equilibria of system (2) are:

1. Zero-contribution: $r_{j}=0, \forall j \in\{1, \ldots, N\}$. This is where no community contributes. We observe that the vector field might not be defined the zero-contribution, otherwise it is an equilibrium.

2. Free-riding equilibria: $\left(0, \ldots, 0, \bar{r}_{j}, 0, \ldots, 0\right)$ for each $j \in$ $\{1, \ldots, N\}$ where $\bar{r}_{j}$ is the aggregated contribution threshold for $j$, i.e. the unique solution $r$ of the equation $v_{j}(r)=1$. These are the $N$ equilibria where only one community contributes to the common good. The other communities may be seen as free-riding on community $j$.

The zero-contribution is always unstable independently if the vector field is defined or not at that point. In fact, in the portion of the coordinate axes (which are invariant) connecting this point to each one of the free-riding equilibria the dynamics goes away from the origin and approaches the corresponding free-riding equilibrium since the marginal utility $\partial \mathcal{U}_{j} / \partial r_{j}$ is positive. This implies that the zero-contribution is indeed a repelling fixed point or a source for the dynamics.

For the other equilibria we will apply Hartman-Grobman's theorem. Consider the equilibrium $\bar{R}_{j}=\left(0, \ldots, 0, \bar{r}_{j}, 0, \ldots, 0\right)$ and let $i \neq j$. So we have:

1. when $k \neq i$ :

$$
\frac{\partial}{\partial r_{k}}\left(\frac{\partial \mathcal{U}_{i}}{\partial r_{i}} r_{i}\right)_{\bar{R}_{j}}=\left(\frac{\partial v_{i}}{\partial r_{k}} r_{i}\right)_{\bar{R}_{j}}=0
$$

2. when $k=i$ :

$$
\frac{\partial}{\partial r_{i}}\left(\frac{\partial \mathcal{U}_{i}}{\partial r_{i}} r_{i}\right)_{\bar{R}_{j}}=v_{i}\left(\bar{r}_{j}\right)-1
$$

Now when $i=j$ we have that for all $k$ :

$\frac{\partial}{\partial r_{k}}\left(\frac{\partial \mathcal{U}_{j}}{\partial r_{j}} r_{j}\right)_{\bar{R}_{j}}=\bar{r}_{j}\left(\frac{\partial v_{j}}{\partial r}\right)_{\bar{R}_{j}}<0$

So the Jacobian matrix at the equilibrium $\bar{R}_{j}=$ $\left(0, \ldots, 0, \bar{r}_{j}, 0, \ldots, 0\right)$ is given by 


$\left[\begin{array}{cccccccc}v_{1}\left(\bar{r}_{j}\right)-1 & 0 & \ldots & 0 & 0 & 0 & \ldots & 0 \\ 0 & v_{2}\left(\bar{r}_{j}\right)-1 & \ldots & 0 & 0 & 0 & \ldots & 0 \\ \vdots & \vdots & \vdots & \vdots & \vdots & \vdots & \vdots & \vdots \\ 0 & 0 & \ldots & v_{j-1}\left(\bar{r}_{j}\right)-1 & 0 & 0 & \ldots & 0 \\ \bar{r}_{j}\left(\frac{\partial v_{j}}{\partial r}\right)_{\bar{R}_{j}} & \bar{r}_{j}\left(\frac{\partial v_{j}}{\partial r}\right)_{\bar{R}_{j}} & \ldots & \bar{r}_{j}\left(\frac{\partial v_{j}}{\partial r}\right)_{\bar{R}_{j}} & \bar{r}_{j}\left(\frac{\partial v_{j}}{\partial r}\right)_{\bar{R}_{j}} & \bar{r}_{j}\left(\frac{\partial v_{j}}{\partial r}\right)_{\bar{R}_{j}} & \ldots & \bar{r}_{j}\left(\frac{\partial v_{j}}{\partial r}\right)_{\bar{R}_{j}} \\ 0 & 0 & \ldots & 0 & 0 & v_{j+1}\left(\bar{r}_{j}\right)-1 & \ldots & 0 \\ \vdots & \vdots & \vdots & \vdots & \vdots & \vdots & \vdots & \vdots \\ 0 & 0 & \ldots & 0 & 0 & 0 & \ldots & v_{N}\left(\bar{r}_{j}\right)-1\end{array}\right]$

It is easy to verify that the matrix has $N$ different eigenvalues which are

$$
\begin{aligned}
v_{1}\left(\bar{r}_{j}\right)-1 & >v_{2}\left(\bar{r}_{j}\right)-1>\ldots>v_{j-1}\left(\bar{r}_{j}\right)-1>v_{j+1}\left(\bar{r}_{j}\right)-1 \\
& >\ldots>v_{N}\left(\bar{r}_{j}\right)-1,
\end{aligned}
$$

and

$\omega=\bar{r}_{j}\left(\frac{\partial v_{j}}{\partial r}\right)_{\bar{R}_{j}}<0$.

The eigenvalue $\omega$ has eigenvector $e_{j}=(0, \ldots, 1, \ldots, 0)$ the $j$ th vector of the canonical basis. The eigenvalue $\lambda_{k}=v\left(\bar{r}_{k}\right)-1$ for $k \neq j$ has eigenvector $v=\omega e_{j}+\left(\lambda_{k}-\omega\right) e_{k}$.

So each free-riding equilibrium has a stable direction along the corresponding coordinate axe. By our previous assumptions we have that $v_{k}\left(\bar{r}_{j}\right)-1>v_{j}\left(\bar{r}_{j}\right)-1=0$ if $k<j$ and that $v_{k}\left(\bar{r}_{j}\right)-1<$ $0=v_{j}\left(\bar{r}_{j}\right)-1$ if $k>j$. Consequently we have that all the steadystates are saddle points with the exception of $\bar{R}_{1}=\left(\bar{r}_{1}, 0, \ldots, 0\right)$, i.e. the equilibria where the only contributing community is the one with the greatest gross marginal benefit for the maintenance of the common good.

Example. For the Baliga-Maskin utility the aggregated contribution threshold for community $j$ is given by $\bar{r}_{j}=\left(\alpha \theta_{j}\right)^{\frac{1}{1-\alpha}}$. Consequently $v_{k}\left(\bar{r}_{j}\right)-1=\theta_{k} / \theta_{j}-1$ which is lesser than zero for $k>j$ and greater than zero for $k<j$. In this case the only asymptotically stable steady-state is $\bar{R}_{1}=\left(\left(\alpha \theta_{1}\right)^{\frac{1}{1-\alpha}}, 0, \ldots, 0\right)$ where only the community with the greatest preference $\theta$ for the common good (in their interpretation the contribution for the reduction of air pollution) is contributing for its maintenance with the others free-riding.

Remark 1. Observe that as we assumed that the costs $\beta_{j}$ are relatively similar, say $\beta_{j} \approx \beta$, then since the thresholds $\bar{r}_{j}$ are all different and ordered according to the marginal gross benefits of the communities, we have that the equilibria of the system are as above, i.e. the zero-contribution and the free-riding equilibria for each community and in order to simplify notation we assumed that $\beta_{j}=1$.

However, if this is not the case and the costs $\beta_{i}$ are not homogeneous, we obtain a similar situation if we substitute the heterogeneity in the gross benefit by heterogeneity in the utilities in the sense that

$\frac{\partial \mathcal{U}_{i}}{\partial r_{i}}>\frac{\partial \mathcal{U}_{i+1}}{\partial r_{i+1}}$

More precisely, in this situation if the marginal utility of community $i$ is zero when the aggregated contribution is $r=\bar{r}_{i}$ then the equilibria are of the above form and the stability considerations that follow also hold. Moreover, this also holds for even more general utility functions $\mathcal{U}_{i}$ with the above property provided they are strictly concave and they are zero for a given aggregated contribution $r=\bar{r}_{i}>0$.
So we have that under general conditions on the utility functions, which are quasi-linear utilities and heterogeneity on the marginal utilities of the communities intervening in the game, the only asymptotically stable equilibrium is the one corresponding to the highest marginal gross benefit. We observe this goes in the sense obtained in [6]: when there is no agreement for the reduction of pollution this is precisely the result of the static individual optimization and the total aggregated contribution is the one corresponding to the community that has the greatest preference for the reduction of air pollution with the other communities freeriding. Our results above assert an identical result in terms of the marginal utilities in a dynamical context of evolution by myopic adaptation.

\section{Conclusion}

In this paper we introduced an evolutionary dynamics for a generalized version of the common good model of Baliga and Maskin (see [6]) and we showed that the only stable equilibrium is the one in which there is a single community willing to contribute for the good which is the one with the highest marginal gross benefit from the maintenance of the good, while all the other communities of the game are free-riding on such contribution, and that all the other equilibria are unstable. Our setting of the problem considers quasi-linear utility functions with marginal gross benefit heterogeneity that includes the Baliga-Maskin environmental game as a particular case. Indeed, this may be seen as reasserting the classic problem of governance of the commons, as for instance presented in [6] in the context of air pollution in an evolutionary framework. Several questions remain. For instance to what extent does the provision of the common good which this single agent can provide is sufficient so that the consumption of that good can be enjoyed by all potential consumers in a sustainable way, i.e. without exhausting it definitively? Or in the context of air pollution, whether the effort of the reduction of pollution by this single agent is sufficient to ensure a sustainable reduction of pollution? Alternatively, how can stable coalition for the maintenance of the common good be formed?

\section{Declaration of Competing Interest}

None.

\section{Acknowledgements}

The authors would like to thank the two anonymous reviewers for their suggestions which greatly improved the paper. This work is partially financed by National Funds through the Portuguese funding agency, FCT - Fundação para a Ciência e a Tecnologia within project UID/EEA/50014/2019, project "Dynamics, optimization and modelling" with reference PTDC/MAT-NAN/6890/2014 and 
the project "Modelling, Dynamics and Games - MDG" with reference PTDC/MAT-APL/31753/2017. Elvio Accinelli thanks the direction of the Economics School of the UASLP for the partial support to develop this work in the Faculty of Sciences of the University of Porto.

\section{References}

[1] Cabrales A. Adaptive dynamics and the implementation problem with complete information. J Econ Theory 1999;86(2):159-84. doi:10.1006/jeth.1999.2530.

[2] Dannenberg A, Lange A, Sturm B. On the Formation of Coalitions to Provide Public Goods - Experimental Evidence from the Lab. NBER Working Papers, 15967. National Bureau of Economic Research, Inc; 2010. https://ideas.repec.org/p/nbr/ nberwo/15967.html.
[3] Hofbauer J, Sigmund K. Adaptive dynamics and evolutionary stability. Appl Math Lett 1990;3(4):75-9. doi:10.1016/0893-9659(90)90051-C.

[4] Killingback T, Bieri J. Flatt T. Evolution in group-structured populations can resolve the tragedy of the commons. Proc Royal Soc B 2006;273(1593):1477-81. doi:10.1098/rspb.2006.3476.

[5] Maskin E. Mechanism design: how to implement social goals. Am Econ Rev 2008;98(3):567-76. http://www.jstor.org/stable/29730086.

[6] Maskin E, Baliga S. Mechanism design for the environment. In: Mäler K, Vincent J, editors. Handbook of environmental economics. Elsevier Science/North Holland; 2003. p. 306-24. doi:10.1016/S1574-0099(03)01012-X.

[7] Olson M. The logic of collective action. Harvard University Press; 2009 (originally published 1965).

[8] Ostrom E. Governing the commons: the evolution of institutions for collective action. Canto classics. Cambridge University Press; 2015. doi:10.1017 CB09781316423936 (originally published 1990).

[9] Sigmund K. The calculus of selfishness. Princeton University Press; 2010. 